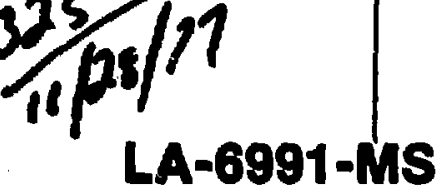

Informal Report
UC-20 and UC-21

Issued: November 1977

\title{
Dependence of Sputtering Erosion on Fuel-Pellet Characteristics
}

\author{
Ihor O. Bohachevsky
}

John F. Hafer 
DEPENDENCE OF SPUTTERING EROSION ON FUEL-PELLET CHARACTERISTICS

by

Ihor 0 . Bohachevsky and John F. Hafer
This neport whs prepared an eccosm of work tponeored by the United Sintes Gormment. Nalihet the United States not the Uniud Stetes Depertment of Exery, nor any of thatr employses, nor any of the contractorh, ubcontrietors, of thate employer, makes

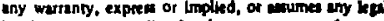
liablity or responsibuty for the secturacy, complatene: or unefulnes of any information, appentus, product of proceses disclosed, of reperients that fits une wothis of infiringe privetely owned righss.

ABSTRACT

Conceptual designs of fusion reactors operating on the principle of inertial confinement require that the dependence of cavity-wall erosion on fuel-pellet onergy yiold, its mass, and representative atomic number be known. A simple approximate model of sputtering erosion is presented and explicit fcmalse are derived that express the total amount of eroded wall chaterial in terms of the above three parameters.

\section{INTRODUCTION}

An approximate model and its analysis are described, which lead to explicit formulae that estimate trends in sputtering erosion without detailed calculations. These results will be useful in parametric studies of conceptual fusion reactor designs operating on the principle of inertial confinewent. For such studies, the dependence of total eroded wall material on the atomic number (Z), of impinging ions on total pellet mass $(M)$, and on its energy yield $(Y)$ must be known. Such knowledge will aid in the selection of desirable materials for structural shells of fuel pellets.

Detailed calculations of sputtering erosion in reactor cavity walls and their application to the conceptual design of fusion reactors have been described previously. ${ }^{1}$ In our present study we approximated the sputtering yield and calculated the erosion explicitly. The model used in the analysis is described, and calculations, results, and conclusions are presented.

\section{THE SPUTTERING MODEL}

The sputtering model contains two parts: (a) the dependence of the sputtering coefficient $S$ on the energy $E$ of impinging lons, and (b) the dependence of ion fluence $N$ on fuel-pellet ass $M$ and on its other charecteristics.

\section{Sputtering Coefficiont S(E)}

To derive the paranetric dependence of sputtefing erosion we postulate that the sputtering coefficient $S$ depends on ion energy $E$ in the following manner.

- $S(E)$ increases linearly from zero at $E=0$ to $S_{m}$ at $E_{m}$, where $S_{m}$ is the maximum value of $s$ and $E_{m}$ is the ion energy at which sputtering is highest;

- $S(E)$ remains constant in the region $E_{m}$ $\leq E \leq r E_{m}$, where $r \geq e$ is an arbitrary constant (the restriction on $r$ is not essential and will be explained later); and

- $\quad S(E)$ decreases as $i n c / e$ for $E=r E_{m}$ where $\epsilon=E / E_{m}$ is the nondimensional ion energy. This behavior, represented schenatically in

Fig. 1, is a reasonable approxination to the theoretically and experimentally determined functional dependence $S(E), 1,2$ In our approximation we assumed that the threshold energy is practically zero, which is satisfactory for our analysis because this energy is usually less than $10 \mathrm{eV}$ and therefore negligible in comparison to $\mathrm{E}_{\mathrm{m}}$, which generally equals tens or hundreds of $k e V$.

Tho physical quantities $E_{m}$ and $s_{m}$ are characteristic of ion material and depend on its atomic number 2; we use Sigmund's theory ${ }^{3}$ to calculate that dependence. The results for molybdenum and carbon targets (walls) are shown in Figs. 2, 3, 4, 


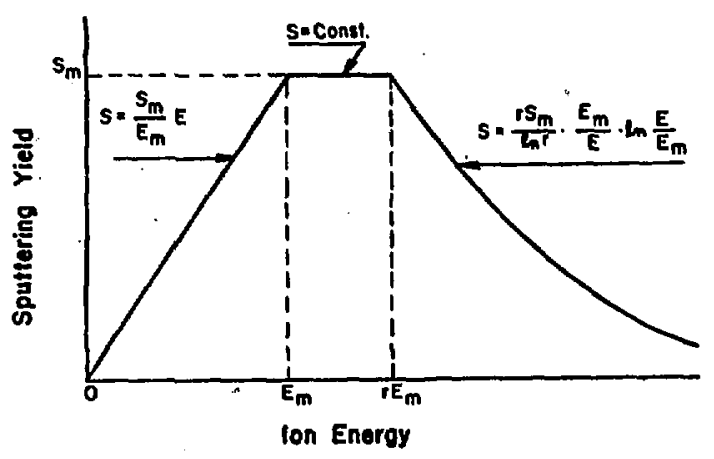

Fig. 1. Model of sputtering yield.

and 5 ; they indicate that $E_{m}(Z)$ is nearly quadratic and $S_{m}(z)$ nearly linear in $z$. Accordingly we set:

$$
\begin{gathered}
E_{m}=K 2^{2} \\
s_{m}=c_{1}+c_{2} z .
\end{gathered}
$$

where the constants $K, C_{1}$, and $C_{2}$ are obtained by matching Eqs. (1) and (2) with theoretically or

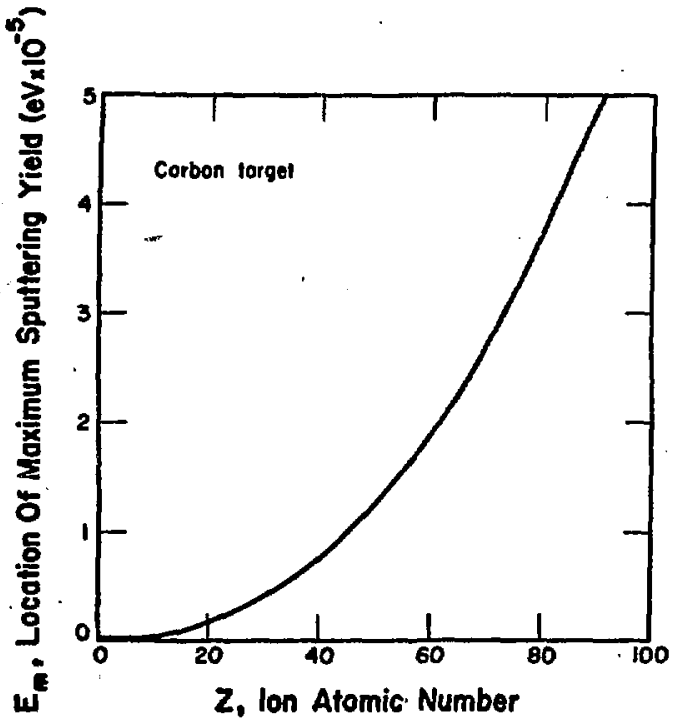

Fig. 3. Maximum of sputtering coefficient for a molybdenum target.

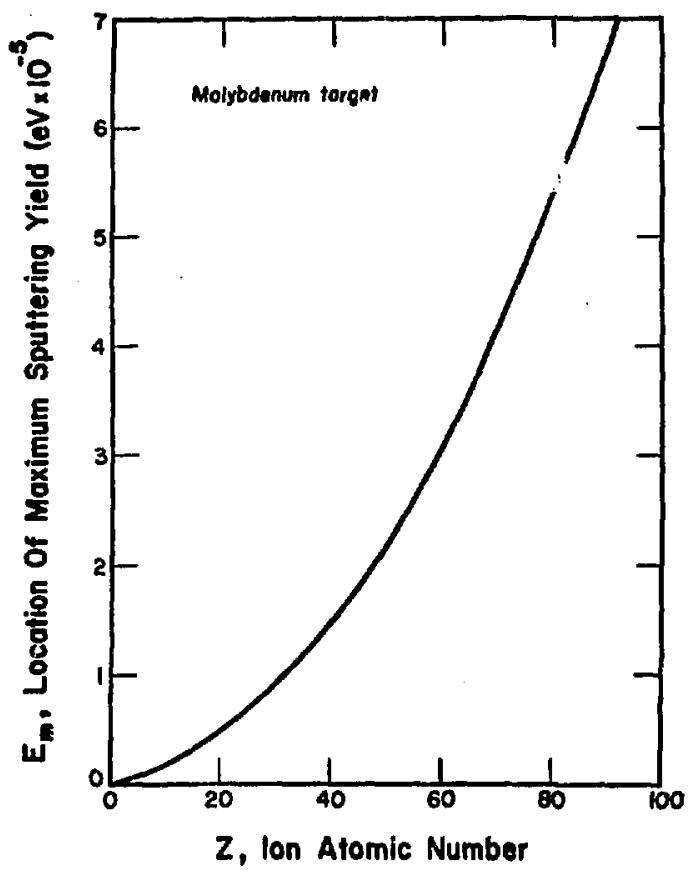
Fig. 2. Location of maximuin sputtering, $S_{m}$,
for a molybdenum target.

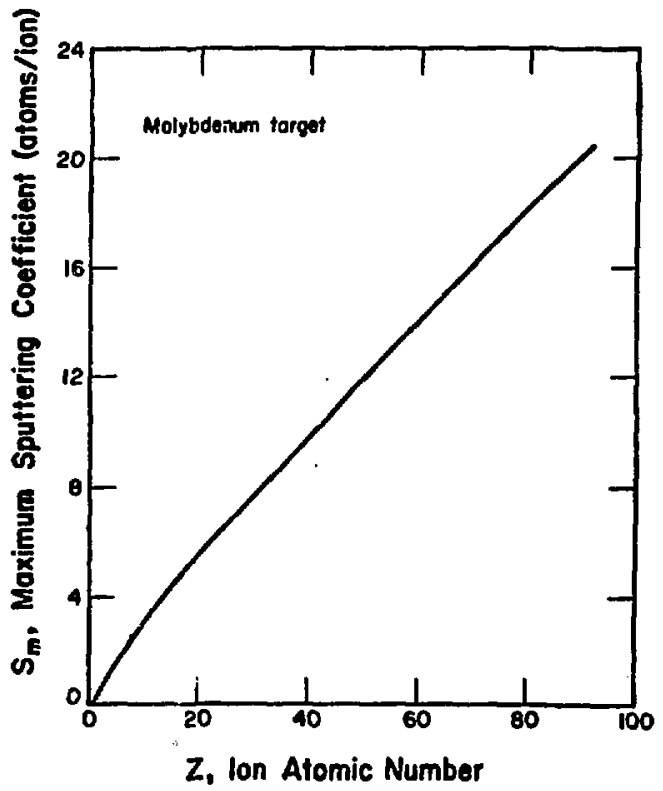

Fig. 4. Location of maximum sputtering, $s_{m}$, for
a carbon target. 


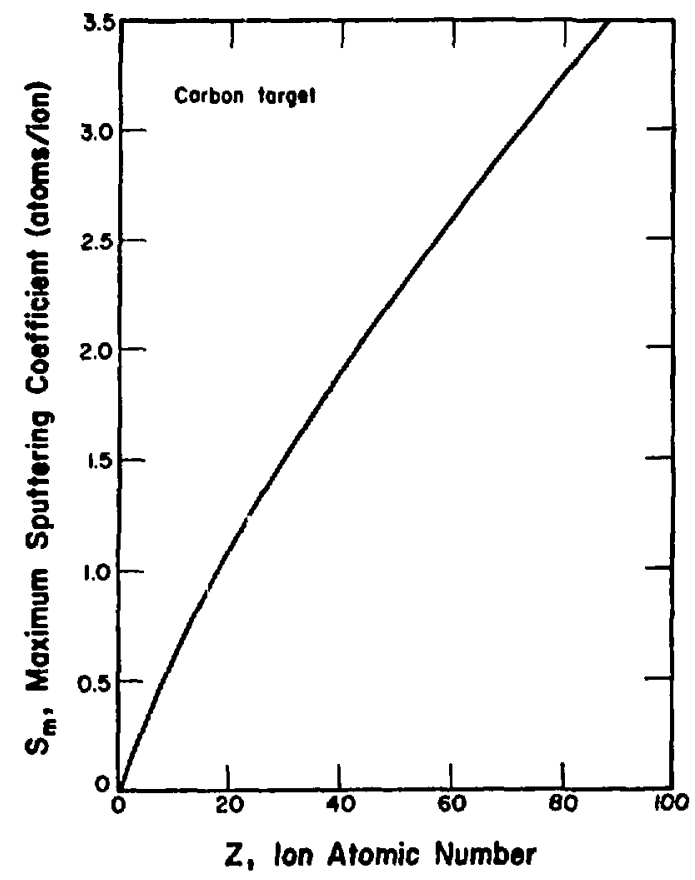

Fig. 5. Maximum of sputtering coefficient for a carbon target.

experimentally obtained dependencies $E_{m}(z)$ and $\mathrm{S}_{\mathrm{m}}(\mathrm{z})$. For example, a molybdenum target (wa11), Figs. 2 and 3 indicate $K \approx 9000, C_{1} \approx 2.0$, and $c_{2} \approx 0.2$.

Consistent with the approximations inherent in Eqs. (1) and (2) we also approximate the atomic weight, $a$, by:

$$
a=2 z+\delta z^{2}
$$

where $\delta$ has a small constant value.

Ion Fluence $\mathrm{N}$

In our parametric analysis we assumed that the pellet composition can be characterized with adequate accuracy by an average atomic weight, $a$. Consequently, the number of plasma ions generated in its microexplosion is:

$$
N=\frac{M A}{a} \text {. }
$$

where $A=6.023 \times 10^{23}$ atoms/mule is Avogadro's number. If we further assume that the yield energy is uniformly distributed over all the ions then the average energy per ton, E, is given by:

$$
E=\frac{f Y_{a}}{M A},
$$

where $Y$ is the total microexplosion yield energy in $e V$, and $f$ is the fraction of yield deposited in plasma debris.

\section{ANALYSIS AND RESULTS}

As stated in the Introduction, the objective of our analysis is the calculation of the total amount of material eroded from the cavity wall of an inertial confinement fusion reactor, given by:

$$
E_{\mathbf{r}}=\mathbf{S} \cdot \mathbf{N}
$$

The three ion-energy regimes--low, intermediate, and high--in which the sputtering coeffictent either increases, remains constant, or decreases will be considered separately.

Low-Energy Regime, $\mathrm{E} \leq \mathrm{E}_{\mathrm{m}}$

Plasma ions will be in the low-energy regine when the inequality ( $\mathrm{Fa} / \mathrm{MA}$ ) $\leq \mathrm{E}_{\mathrm{m}}$ is satisfied; in general, this is true when the pellet yield-tomass ratio i's low.

In this regime sputtering yield increases linearly with ion energy and therefore is given by $S=\left(S_{m} / E_{m}\right) E$. Using this expression together with Eqs. (4) and (5) in Eq. (6) we obtain

$$
E_{r}=\frac{S_{m}}{E_{m}} f Y,
$$

which upon substitution of expressions for $E_{\text {in }}$ and $S_{m}$ [Eqs. (1) and (2), respectively] becomes

$$
E_{\mathbf{r}}=\frac{C_{1}+c_{2} Z}{k z^{2}} f Y
$$

This result shows that in the low-energy ragime (where the sputtering coefficient increases with ion energy) the total amount of material eroded is independent of pellet mass; is directly proportional to the total ion energy; and, for a constant yield, decreases with increasing atomic number, $Z$, of the pellet material. 
Intermediate-Energy Regime, $E_{m} \leq E \leq r E_{m}$

In this regime, the Ion energy satisfies the inequalities $E_{m}<\left(f Y_{a}\right) /(M A) \leq r E_{m}$ and the sputtering coefficient does not change with ion onergy. Consequently, the amount of eroded material is given by:

$$
E_{r}=S_{m} \frac{M A}{a}
$$

By using Eqs. (2) and (3) to express $E_{T}$ in terms of atomic number wo obtain:

$$
E_{r}=\left(\frac{M A}{Z}\right) \frac{C_{1}+C_{2} Z}{2+6 Z} \text {. }
$$

Thi, result shows that in the intermediateenergy ragime (where the sputtering coefficient is Independent of ion energy) the anount of eroded material does not directly depend on fuel-pellet energy yield; is proportional to pellet mass, $M$; and for constant $M$ decreases with increasing $Z$. The decrease, however, may not be uniform for some combinations of values for $C_{1}, C_{2}$, and $\delta$. High-Energy Regime, $\mathrm{E}>\mathrm{rE}_{\mathrm{m}}$

Plasma ions are in the high-energy regime when the inequality ( $\mathrm{fYa} / \mathrm{MA}$ ) $>r E_{m}$ is satisfied; in genoral this is true when the pellet yield-tomass ratio is high.

Theoretical and experimental investigations indicate ${ }^{2,3}$ that in the high-energy regime the sputtering coefficient decreases with increasing energy as $\epsilon^{-1}$ ine--(this function decreases monoconically for $\epsilon \geq e$, hence the provious restriction on $r$ ); consequently:

$$
S=\frac{T}{\ln r} \frac{\ell n e}{E},
$$

where the uultiplicative constant was determined from the continuity of $S$ at $E=r E_{n}$.

Substituting the above expression for $S$ together with Eqs. (4) and (5) into Eq. (6) we obtain:

$$
E_{r}=\frac{r(M A)^{2}}{f Y \ln r} \frac{E_{m} S_{m}}{a^{2}} \ln \frac{f Y a}{M A E_{m}}
$$

In this expression the product Es increases as $z^{3}$. [Eqs. (1) and (2)] and $a^{2}$ increases as $z^{2}$
[Eq. (3)]. However, no convenient estimate exists that would reflect the variation of the logarithmic term with 2 . The following considerations indicate the behavior of $E_{r}$. For low $Z$ values $E_{m}$ is relatively small making the argument of the logarithm large and the logarithm itself slowly varying with 2 . Thus the behavior of $\mathrm{E}_{\mathbf{T}}$ is determined predominantly by the factor $E_{m} S_{m} / a^{2}$, which increases with 2 . For high values of $Z$ the argunent of the logarithm decreases as a constant divided by $Z$ and the logarithm itself diminishes more rapidly than the increasing factor $E_{m} s_{m} / a^{2}$. Thus, $E_{T}$ eventually begins to decrease with increasing $\mathrm{Z}$.

To verify the above deduced behavior and to obtain an idea of the value of $Z$ at which $E_{r}$. changes from an increasing to a decreasing function of $Z$ we havo evaluated Expression (9) numerically by using exact expresstons from Sigmund's theory for $E_{m}(Z)$ and $s_{m}(Z), 3$ and exact values from the periodic table of elements for $a(z)$. The calculation was carried out fox $r=2.80, f=0.20$, $Y=100 \mathrm{MJ}$, and $M=0.07 \mathrm{~g}$ chosen so that ions are in the high-energy regime for all values of $z \leq 48$.

The result is plotted in Fig. 6 . It shows that $E_{r}$ behaves as predicted: Initially increases with 2 , reaches a maximum (at $Z=8$ for the particular set of parameter values used in' this sample computation), and then decreases as $Z$ increases. The curve is extended beyond $Z=49$ to illustrate the trend. The behavior, however, is not uniformly smooth but shows small undulations in the general trend near the maximun.

\section{CLOSING COMNENTS}

The analysis presented in this report led to expressions that estimate the total anount of eroded wall material for different impinging ions in terms of ion atomic number and other parameters characterizing ion fluence. The complewentary problew of determining amounts of different wall materials eroded by a given ion flux has becn investigated extensively by Wehner and his collaboraters .4,5,6 Their findings are sumarized in Sec. 7.4.2 of Ref. 2; the results presented in convenient graphical form may be found on pp. 315316 and need not be reproduced here. Briefly, the 


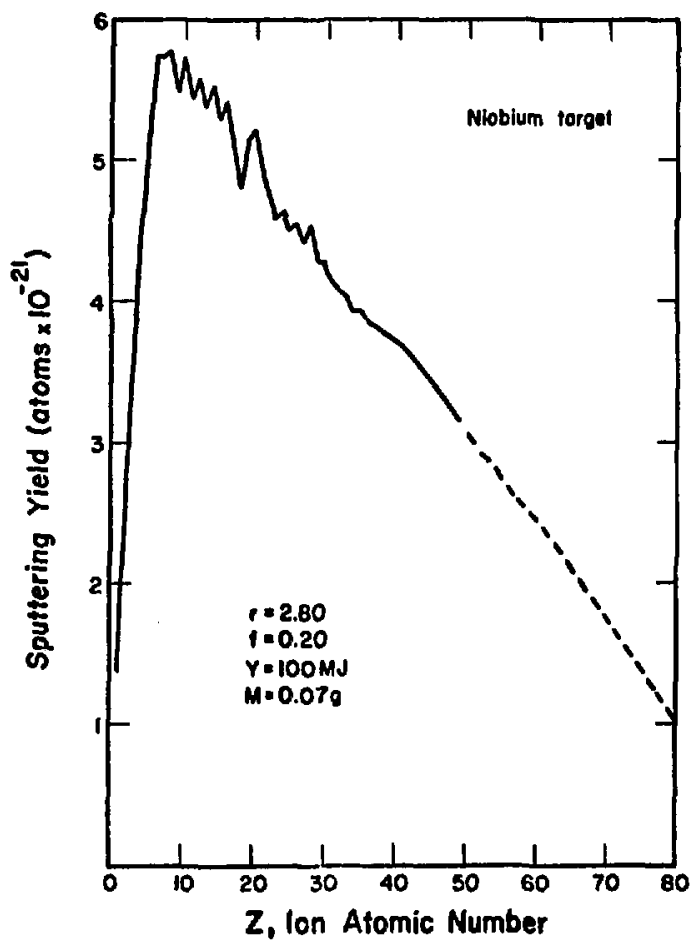

Fig. 6. Total sputtering erosion in the highion-energy regime. sputtering yield follows closely, with minor exceptions, the state of electron concentrations in the " $d$ " shells of target (wall) material atoms and also crudely resembles the reciprocals of the heats of siblimation. Thus the yield varies almost periodically with target atomic number.

ACKNOWLEDGMENTS

Constructive suggestions by J. J. Devaney and T. G. Frank are gratefully acknowledged.

\section{REFERENCES}

1. I. 0. Bohachevsky and J. F. Hafer, "Sputtering Erosion of Reactor Cavity Walls," Los Alamos Scientific Laboratory report LA-6633MS (in process).

2. G. Carter and J, S. Colligon, Ion Bombarciment of Solids (American Elsevier Publishing Company, New York, 1968).

3. P. Sigmund, "Theory of Sputtering I. Sputtering Yield of Amorphous and Polycrystalline Tergets," Phys. Rev. 184, 383 (August 1969).

4. G. K. Wehnef, "Sputtering Yields for Norma1ly Incident $\mathrm{Hg}$-Ion Bombardment at Low Ion Energy," Phys. Rev. 108, 35 (1957).

5. N. Laegreid and G. K. Węhner, "Şputtering Yields of Metals for $\mathrm{Ar}^{+}$and $\mathrm{Ne}$ Ions with Energies from 50 to $600 \mathrm{eV."} \mathrm{J.} \mathrm{Appl.} \mathrm{Phys.,}$ 32, 365 (March 1961).

6. N. Laegreid and S. D. Dahlgren, "Wall Splicicer ing and Wall Life," J. Appl. Phys., A4, 2093 (May 1973). 